\title{
Undefinability in Inquisitive Logic with Tensor
}

\section{Ciardelli, Ivano}

Springer

2019

Ciardelli , I \& Barbero , F 2019 , Undefinability in Inquisitive Logic with Tensor . in P Blackburn, E Lorini \& M Guo (eds), Logic, Rationality and Interaction : 7th International pÿWorkshop, LORI 2019, Chongqing, China, October 18 21, 2019, Proceedings . Lecture Notes in Computer Science edn, vol. 11813 , Lecture Notes in Computer Science, vol. 11813 , Springer , pp. 29-42, 7th International Workshop, LORI 2019 , Chongqing , China , 18/10/2019 . https://doi.org/10.1007/978-3-662-60292-8_3

http://hdl.handle.net/10138/310282

https://doi.org/10.1007/978-3-662-60292-8_3

unspecified

acceptedVersion

Downloaded from Helda, University of Helsinki institutional repository.

This is an electronic reprint of the original article.

This reprint may differ from the original in pagination and typographic detail.

Please cite the original version. 


\title{
Undefinability in inquisitive logic with tensor
}

\author{
Ivano Ciardelli ${ }^{10000-0001-6152-3401]}$ and Fausto Barbero ${ }^{20000-0002-0959-6977]}$ \\ 1 Munich Center for Mathematical Philosophy, LMU Munich, Germany \\ ivano.ciardelli@lrz.uni-muenchen.de \\ 2 Department of Philosophy, University of Helsinki, Finland \\ fausto.barbero@helsinki.fi
}

\begin{abstract}
Logics based on team semantics, such as inquisitive logic and dependence logic, are not closed under uniform substitution. This leads to an interesting separation between expressive power and definability: it may be that an operator $\mathrm{O}$ can be added to a language without a gain in expressive power, yet $\mathrm{O}$ is not definable in that language. For instance, even though propositional inquisitive logic and propositional dependence logic have the same expressive power, inquisitive disjunction and implication are not definable in propositional dependence logic. A question that has been open for some time in this area is whether the tensor disjunction used in propositional dependence logic is definable in inquisitive logic. We settle this question in the negative. In fact, we show that extending the logical repertoire of inquisitive logic by means of tensor disjunction leads to an independent set of connectives; that is, no connective in the resulting logic is definable in terms of the others.
\end{abstract}

Keywords: definability · inquisitive logic · tensor disjunction · dependence logic $\cdot$ team semantics $\cdot$ conjunction

\section{Introduction}

The notion of definability of a connective in terms of a set of other connectives is one of the basic notions of propositional logic. In classical logic every truth-functional connective is definable from, say, the set $\{\neg, \wedge\}$; for instance, disjunction can be defined as $\varphi \vee \psi:=\neg(\neg \varphi \wedge \neg \psi)$ and implication as $\varphi \rightarrow \psi:=\neg(\varphi \wedge \neg \psi)$. By contrast, it was shown by McKinsey [18] that in intuitionistic logic none of the primitive connectives $\neg, \wedge, \rightarrow, \vee$ whose semantics is characterized by the BHK interpretation is definable in terms of the other. In the setting of classical and intuitionistic logic, questions of definability are tighly connected to questions about the expressive power of a certain repertoire of connectives. If a connective $\circ$ is definable from other connectives, then each occurrence of this connective can be paraphrased away according to its definition; therefore, the connective can be omitted from the language without loss of expressive power. Conversely, if o can be eliminated from the language without loss of expressive power, then $\circ$ can also be defined. To see why, suppose for concreteness that $\circ$ is binary. If $\circ$ can be omitted from the language, then in particular the formula $p \circ q$, where $p$ and $q$ are atomic formulas, is equivalent to 
some formula $\varphi(p, q)$ which does not contain $\circ$. In both classical and intuitionistic logic, logical equivalences remain valid when we replace atoms by arbitrary formulas. Therefore, we also have that for all $\psi$ and $\chi, \psi \circ \chi$ is equivalent to $\varphi(\psi, \chi)$. This means that the formula $\varphi(p, q)$, when viewed as a scheme where $p$ and $q$ are placeholders for the two arguments, provides a definition of the connective $\circ$.

This connection between definability and expressive power holds because of an important property of classical and intuitionistic logic, namely, closure under uniform substitution: logical equivalences remain valid when we replace atomic formulas by arbitrary formulas. In the recent literature, a number of logics have been studied which lack this property: in these logics, atomic formulas are not viewed as placeholders for arbitrary sentences, but rather as placeholders for sentences of a particular kind, which may have special logical properties. Two families of non substitution-closed logics which have been investigated thoroughly in the last few years are inquisitive logic (see, e.g., $[8,21,20,6,14,7])$ and dependence logic (see, e.g., $[22,1,17,11,12,26,28]){ }^{3}$ These approaches arose independently, and from different enterprises: inquisitive logic is concerned with extending the scope of logic to questions, while dependence logic is concerned with enriching classical logic with formulas that talk about dependencies and independencies between variables. However, these two approaches turned out to be tightly connected from a mathematical perspective $[26,5,28]$; and the convergence is not accidental: as discussed in $[5,7]$ the relation of dependency can be seen as a special case of the relation of entailment, once the latter is generalized to questions. In the propositional setting, the standard incarnation of these two approaches is given by the systems of propositional inquisitive logic, InqB [8], and propositional dependence logic, PD [28]. These systems are interpreted in the same semantic setting, namely, in terms of a relation $\models$ which is assessed relative to sets of propositional valuations. However, these systems differ in their repertoire of logical operation. The set of primitives in InqB comprises the constant $\perp$ and the binary connectives $\wedge$ (conjunction), $\rightarrow$ (inquisitive implication) and $\mathbb{V}$ (inquisitive disjunction); from these operators negation is defined as $\neg \varphi:=\varphi \rightarrow \perp$. The logical repertoire of PD comprises the connectives $\wedge$ (conjunction, the same operator as in InqB) and $\otimes$ (tensor disjunction) as well as two operators that can be applied only to atomic formulas: negation $\neg$, and the dependence atom $=(;)$ which is an operator of variable arity, taking an arbitrary sequence of atoms in the first coordinate and an atom in the second.

InqB, PD, and their extensions are not closed under uniform substitution. As a consequence, in these logics expressive power and definability come apart. It is quite possible that a connective $\circ$ can be dropped from the language without loss of expressive power, yet this connective is not definable from the remaining connectives. This happens when each particular occurrence of o can be paraphrased away, but the required paraphrase depends on the specific arguments to which $\circ$

\footnotetext{
${ }^{3}$ Other examples of non-substitution closed logics are data logic [23], several dynamic epistemic logics $[19,2,10]$, as well as logics arising from dynamic semantics $[9,24,15]$ and from expressivist approaches to modals and conditionals $[25,16,3]$.
} 
applies. For instance, it was shown in [4] that every formula of InqB is equivalent to one in the language $\{\neg, \mathbb{V}\}$, and also to one in the language $\{\perp, \rightarrow, \mathbb{V}\}$; yet, implication is not definable in terms of $\{\neg, \mathbb{V}\}$, and conjunction is not definable in terms of either $\{\neg, \mathbb{V}\}$ or $\{\perp, \rightarrow, \wedge\}$.

More recently, [28] showed that PD has the same expressive power as InqB. Adding the inquisitive connectives $\mathbb{V}$ and $\rightarrow$ to the logical repertoire of PD would not increase the expressive power of the language. However, one may still ask whether these operators are definable in PD. As shown in [27], the answer is negative: both $\rightarrow$ and $\mathbb{V}$ are not definable in PD. ${ }^{4}$ Similarly, adding the dependence logic operators $=(;)$ and $\otimes$ to the inquisitive repertoire does not increase the expressive power of InqB. But are these operators definable in InqB? For the dependence atom, the answer is easy: it is definable in InqB by means of the inquisitive connectives by the following scheme:

$$
=\left(p_{1}, \ldots, p_{n} ; q\right):=? p_{1} \wedge \cdots \wedge ? p_{n} \rightarrow ? q
$$

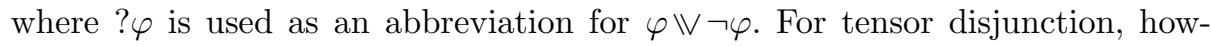
ever, the question has so far remained open. It was conjectured in [27] and [6] that $\otimes$ is not definable from the inquisitive connectives, but a proof has so far been missing. Our main aim in this paper is to prove this conjecture. In fact, we will take this opportunity to investigate more thoroughly matters of expressive power and definability in the context of inquisitive logic extended with the tensor disjunction connective. We will show that adding $\otimes$ to InqB leads to an independent set of primitives: that is, not only is $\otimes$ not definable from the inquisitive connectives, but none of the inquisitive connectives becomes definable from the other inquisitive connectives in the presence of tensor disjunction.

The paper is structured as follows. In Section 2 we specify the notions of definability and eliminability of a connective and discuss the relations between the two. In Section 3 we define $\operatorname{lnq} B^{\otimes}$, inquisitive logic with tensor, and mention some key properties of this logic. In Section 4 we look into expressive completeness: we show that, starting from the repertoire $\{\perp, \wedge, \rightarrow, \mathbb{V}, \otimes\}$, there is a unique minimal set of connectives which is expressively complete, namely, $\{\perp, \rightarrow, \mathbb{V}\}$; thus, $\wedge$ and $\otimes$ can be removed from the language without loss of expressive power. In Section 5 we look into definability, showing that no connective in $\{\perp, \wedge, \rightarrow, \mathbb{V}, \otimes\}$ is definable from the others; the novel results are the undefinability of tensor disjunction from the inquisitive connectives, and the undefinability of conjunction from $\{\perp, \rightarrow, \mathbb{V}, \otimes\}$. Section 6 concludes.

\footnotetext{
${ }^{4}$ For other undefinability results in the setting of dependence logic, see also $[12,13]$. It is worth noting that, in the dependence logic literature, the standard notion of definability is called uniform definability; since there seems to be no special reason to add the qualification uniform (the notion of definability is intrinsically "uniform" in the relevant sense) we prefer to stick with the standard terminology.
} 


\section{Definability and eliminability}

Throughout this section, let $L$ be an arbitrary propositional logic with language $\mathcal{L}=\mathcal{L}[C]$ generated by a set $C$ of connectives, giving rise to a relation of logical equivalence $\equiv_{L} \subseteq \mathcal{L} \times \mathcal{L}$. We assume $\equiv_{L}$ to be an equivalence relation and a congruence with respect to the connectives: that is, we suppose that for every $n$-ary connective $\circ$, if $\varphi_{i} \equiv_{L} \psi_{i}$ for $i \leq n$ then $\circ\left(\varphi_{1}, \ldots, \varphi_{n}\right) \equiv_{L} \circ\left(\psi_{1}, \ldots, \psi_{n}\right)$.

Definition 1 (Context). A propositional context $\varphi\left(p_{1}, \ldots, p_{n}\right)$ consists of a formula $\varphi \in \mathcal{L}$ together with a sequence of designated atomic formulas $p_{1}, \ldots, p_{n}$. Note that $\varphi\left(p_{1}, \ldots, p_{n}\right)$ is allowed to contain other atoms besides $p_{1}, \ldots, p_{n}$. If $\varphi\left(p_{1}, \ldots, p_{n}\right)$ is a context and $\chi_{1}, \ldots, \chi_{n} \in \mathcal{L}$, we write $\varphi\left(\chi_{1}, \ldots, \chi_{n}\right)$ for the result of replacing $p_{1}, \ldots, p_{n}$ by $\chi_{1}, \ldots, \chi_{n}$ throughout $\varphi$.

Definition 2 (Definability). We say that an n-ary connective $\circ \in C$ is defined by a context $\xi\left(p_{1}, \ldots, p_{n}\right)$ in case for all $\chi_{1}, \ldots, \chi_{n} \in \mathcal{L}[C]$ :

$$
\circ\left(\chi_{1}, \ldots, \chi_{n}\right) \equiv_{L} \xi\left(\chi_{1}, \ldots, \chi_{n}\right)
$$

We say that $\circ$ is definable from a set $C^{\prime} \subseteq C$ of connectives in case there is a context $\xi\left(p_{1}, \ldots, p_{n}\right)$, with $\xi \in \mathcal{L}\left[C^{\prime}\right]$ which defines $\circ$. If we just say that $\circ$ is definable then we mean that it is definable from $C-\{0\}$.

In terms of definability we define the notion of an independent set of connectives.

Definition 3 (Independence). We say that a set $C^{\prime}$ of connectives is independent if no connective $\circ \in C^{\prime}$ is definable from $C^{\prime}-\{0\}$.

In addition to the notion of definability, we also introduce a notion of eliminability of a connective, which means that the connective can be omitted from the language without a loss in expressive power.

Definition 4 (Eliminability). Let $C^{\prime} \subseteq C$ be a set of connectives. We say that the set of connectives $C^{\prime}$ is eliminable if for each formula $\varphi \in \mathcal{L}[C]$ there is a formula $\varphi^{*} \in \mathcal{L}\left[C-C^{\prime}\right]$ such that $\varphi \equiv_{L} \varphi^{*}$. We say that a connective $\circ$ is eliminable if $\{0\}$ is eliminable.

Notice that definability implies eliminability.

Proposition 1. If a connective $\circ$ is definable, then it is eliminable.

Proof. Suppose $\circ$ is defined by $\xi\left(p_{1}, \ldots, p_{n}\right) \in \mathcal{L}[C-\{\circ\}]$. We show by induction that every $\varphi \in \mathcal{L}[C]$ is equivalent to some $\varphi^{*} \in \mathcal{L}[C-\{0\}]$. The only nontrivial case is the one for $\varphi=\circ\left(\psi_{1}, \ldots, \psi_{n}\right)$. By induction hypothesis there are $\psi_{1}^{*}, \ldots, \psi_{n}^{*} \in \mathcal{L}[C-\{\circ\}]$ s.t. $\psi_{i} \equiv_{L} \psi_{i}^{*}$ for $i \leq n$. Then $\varphi \equiv_{L} \circ\left(\psi_{1}^{*}, \ldots, \psi_{n}^{*}\right)$. Since $\circ$ is defined by $\xi$ we have $\circ\left(\psi_{1}^{*}, \ldots, \psi_{n}^{*}\right) \equiv_{L} \xi\left(\psi_{1}^{*}, \ldots, \psi_{n}^{*}\right)$. Since $\xi, \psi_{1}^{*}, \ldots, \psi_{n}^{*} \in$ $\mathcal{L}[C-\{\circ\}]$, we have that $\xi\left(\psi_{1}^{*}, \ldots, \psi_{n}^{*}\right) \in \mathcal{L}[C-\{\circ\}]$. So $\varphi$ is $L$-equivalent to some formula in $\mathcal{L}[C-\{\circ\}]$, which completes the inductive step. 
Another important notion in this area is the notion of completeness of a set $C^{\prime}$ of connectives, which holds when every formula in the language is $L$-equivalent to one built up using only connectives from $C^{\prime}$.

Definition 5 (Completeness). We say that a set of connectives $C^{\prime} \subseteq C$ is complete for $L$ if for all $\varphi \in \mathcal{L}[C]$ there exists some $\varphi^{*} \in \mathcal{L}\left[C^{\prime}\right]$ s.t. $\varphi \equiv_{L} \varphi^{*}$. $W e$ say that a set $C^{\prime}$ is a minimal complete set of connectives for $L$ if $C^{\prime}$ is complete for $L$, and no proper subset $C^{\prime \prime} \subset C^{\prime}$ is complete for $L$.

The notions of definability and eliminability often go hand in hand. This is because the $\operatorname{logics} L$ one typically considers are closed under uniform substitution. Closure under uniform substitution is usually formulated in terms of preservation of validity under substitutions. For our purposes, a characterization in terms of preservation of equivalence is more suitable. The two coincide if $L$ validates a deduction theorem, and more generally if $\varphi \equiv_{L} \psi$ amounts to the validity of a formula $\chi(\varphi, \psi)$ in $L$ (if the deduction theorem holds, $\chi(\varphi, \psi)$ will be $\varphi \leftrightarrow \psi$ ).

Definition 6 (Closure under uniform substitution). $L$ is closed under uniform substitution if for any two contexts $\varphi\left(p_{1}, \ldots, p_{n}\right)$ and $\psi\left(p_{1}, \ldots, p_{n}\right)$, if $\varphi \equiv_{L} \psi$ then for all $\chi_{1}, \ldots, \chi_{n} \in \mathcal{L}$ we have $\varphi\left(\chi_{1}, \ldots, \chi_{n}\right) \equiv_{L} \psi\left(\chi_{1}, \ldots, \chi_{n}\right)$.

In a logic which is closed under uniform substitution, the notions of definability and eliminability for a connective coincide.

Proposition 2. If $L$ is closed under uniform substitution and $\circ$ is eliminable, then $\circ$ is definable.

Proof. Let $p_{1}, \ldots, p_{n}$ be $n$ distinct atomic formulas. Suppose $L$ is closed under uniform substitution and $\circ$ is eliminable. Then the formula $\circ\left(p_{1}, \ldots, p_{n}\right)$ is $L$ equivalent to some $\xi \in \mathcal{L}[C-\{\circ\}]$. Consider the context $\xi\left(p_{1}, \ldots, p_{n}\right)$ : by closure under uniform substitution, for all $\chi_{1}, \ldots, \chi_{n} \in \mathcal{L}$ we have $\circ\left(\chi_{1}, \ldots, \chi_{n}\right) \equiv_{L}$ $\xi\left(\chi_{1}, \ldots, \chi_{n}\right)$. Thus, $\circ$ is defined by $\xi\left(p_{1}, \ldots, p_{n}\right)$.

As we discussed in the introduction, systems of inquisitive and dependence logic are typically not closed under uniform substitution. As a consequence, in these logics we find an interesting gap between the eliminability of a connective and its definability. We will examine this gap carefully for one particular logic, the system $\operatorname{lnqB}{ }^{\otimes}$ introduced in the next section.

\section{Inquisitive logic with tensor disjunction}

In this section we introduce the system $\operatorname{lnq} B^{\otimes}$ that we will be concerned with. This system extends the standard system of propositional inquisitive logic, InqB $[4,8,6]$ with the tensor disjunction used in dependence logic $[26,28]$. We will present this logic from a purely mathematical point of view. For an introduction to the motivations of inquisitive logic and to the conceptual interpretation of formulas in this system, see $[6,7]$. 
Formally, the language $\mathcal{L}$ of $\operatorname{lnqB}^{\otimes}$ is generated by the set of connectives $\{\perp, \wedge, \mathbb{V}, \rightarrow, \otimes\}$, where all connectives are binary except for $\perp$, which has arity 0 :

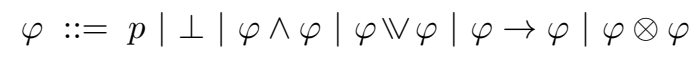

In addition to the primitive connectives, some defined connectives are used:

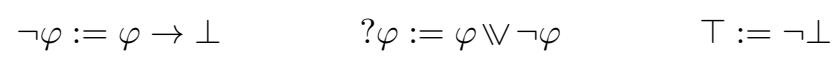

In classical propositional logic, the semantics of a formula is given in terms of truth-conditions relative to a valuation function $w: \mathcal{P} \rightarrow\{0,1\}$, which represents a complete state of affairs. By contrast, in inquisitive logic, formulas are interpreted relative to a set of such valuation functions. Informally, such a set $s$ is taken to represent an information state, where the idea is that $s$ embodies the information that the actual state of affairs corresponds to one of the valuations $w \in s$; thus, the smaller the set $s$, the stronger the information it encodes.

Definition 7 (Information states).

An information state is a set $s$ of valuations for $\mathcal{P}$, i.e., a subset $s \subseteq\{0,1\}^{\mathcal{P}}$.

The semantics is given in terms of a relation of support relative to information states, which is defined inductively as follows.

\section{Definition 8 (Support).}

$-s=p \Longleftrightarrow w(p)=1$ for all $w \in s$

$-s \models \perp \Longleftrightarrow s=\emptyset$

$-s=\psi \wedge \chi \Longleftrightarrow s \models \psi$ and $s=\chi$

$-s \models \psi \vee \chi \Longleftrightarrow s \models \psi$ or $s \models \chi$

$-s \models \psi \rightarrow \chi \Longleftrightarrow \forall t \subseteq s: t=\psi$ implies $t \models \chi$

$-s \models \psi \otimes \chi \Longleftrightarrow \exists t_{1}, t_{2}$ s.t. $t_{1} \models \psi, t_{2} \models \chi$ and $s=t_{1} \cup t_{2}$.

It is immediate to see that the defined operators have the following semantics:

$-s=\neg \psi \Longleftrightarrow \forall t \subseteq s: t=\psi$ implies $t=\emptyset$

$-s=? \psi \Longleftrightarrow s \models \psi$ or $s \models \neg \psi$

$-s \models \top$ for all information states $s$.

The support relation has the following properties.

- Persistency: if $s \models \varphi$ and $t \subseteq s$, then $t \models \varphi$

- Empty state property: $\emptyset \models \varphi$ for all $\varphi$.

From the notion of support at a state, a notion of truth relative to a valuation $w$ is recovered by defining truth at $w$ as support with respect to $\{w\}$.

Definition 9 (Truth). We say that $\varphi$ is true at $w$, notation $w \models \varphi$, if and only if $\{w\} \models \varphi$. 
It is easy to see that the truth-conditions that this delivers are simply the ones familiar from classical propositional logic, when both $\mathbb{V}$ and $\otimes$ are identified with classical disjunction $\vee$. Notice that, due to persistency, if a formula $\varphi$ is supported at $s$, then it is true at all valuations $w \in s$. The converse is not true in general, but it may hold for particular formulas. The formulas for which this is the case are called truth-conditional, since the semantics of these formulas is completely determined at the level of truth-conditions. ${ }^{5}$

Definition 10 (Truth-conditionality). We say that $\varphi \in \mathcal{L}$ is truth-conditional if for all information states $s$ we have: $s \models \varphi \Longleftrightarrow \forall w \in s: w \models \varphi$

There is an important fragment of our language which consists entirely of truthconditional formulas. We refer to the formulas in this fragment as declaratives.

Definition 11 (Declaratives). A formula $\varphi \in \mathcal{L}$ is a declarative if it is $\mathbb{V}$ free. In other words, the set of declaratives is the set $\mathcal{L}_{!}:=\mathcal{L}[\perp, \wedge, \rightarrow, \otimes]$.

Proposition 3 (cf. [5], Prop. 3). Every $\alpha \in \mathcal{L}_{!}$is truth-conditional.

Formulas containing $\mathbb{V}$ are not in general truth-conditional. For instance, consider ? $p$ (which abbreviates $p \llbracket \neg p$ ). Since truth-conditions coincide with truthconditions in classical propositional logic, ? $p$ will be true with respect to all valuations $w$ whatsoever. However, the support-conditions for this formula are:

$s \models ? p \Longleftrightarrow s \models p$ or $s \models \neg p \Longleftrightarrow(\forall w \in s: w(p)=1)$ or $(\forall w \in s: w(p)=0)$

where the last equivalence uses the fact that $p$ and $\neg p$ are declaratives, and thus truth-conditional. Thus, ? $p$ is supported at a state $s$ only if all the valuations in $s$ agree about the truth-value of $p$. Thus, if $s$ is a state containing some valuations that make $p$ true and some that make $p$ false, then $s \not \models ? p$, even though ? $p$ is true at all $w \in s$. This shows that ?p is not truth-conditional.

Logical entailment and equivalence are defined naturally in terms of support.

Definition 12 (Logical entailment and equivalence). We say that:

$-\varphi$ entails $\psi, \varphi \models \psi$, if for all states $s: s \mid=\varphi$ implies $s \models \psi$;

$-\varphi$ and $\psi$ are equivalent, $\varphi \equiv \psi$, if for all states $s: s \models \varphi$ iff $s=\psi$.

In addition to these purely logical notions, it will also be useful to have notions of entailment and equivalence relativized to an information state $s$. The idea is that, when looking at entailment and equivalence relative to $s$, only valuations in $s$ are taken into account (for discussion of the significance of logical and contextual entailment in inquisitive logic, see [6]§1 and [7]).

Definition 13 (Relativized entailment and equivalence). Let $\varphi, \psi \in \mathcal{L}$ and let $s$ be an information state. We say that:

$-\varphi$ entails $\psi$ in $s, \varphi \models_{s} \psi$, if for all states $t \subseteq s: t \models \varphi$ implies $t \models \psi$;

\footnotetext{
${ }_{5}$ Truth-conditional formulas are called flat formulas in the dependence logic literature.
} 
$-\varphi$ and $\psi$ are equivalent in $s, \varphi \equiv_{s} \psi$, if for all states $t \subseteq s: t \models \varphi$ iff $t \models \psi$.

Notice that logical entailment implies entailment relative to any information state; similarly, logical equivalence implies equivalence relative to any state.

For formulas in the declarative fragment of the language, logical entailment and equivalence coincide with entailment and equivalence in classical propositional logic, when the operator $\otimes$ is identified with classical disjunction. We state the case of equivalence as a proposition, since we will make use of it below.

Proposition 4 (cf. [5], Prop. 6).

For all $\alpha, \beta \in \mathcal{L}_{!}: \alpha \equiv \beta \Longleftrightarrow \alpha$ and $\beta$ are equivalent in classical propositional logic, when $\otimes$ is replaced by the classical disjunction symbol $\vee$.

An important feature of propositional inquisitive logic, which extends to $\operatorname{lnq} \mathrm{B}^{\otimes}$ [5], is that every formula is equivalent to an inquisitive disjunction of declaratives.

Theorem 1 (cf. [5], Prop. 11). For every $\varphi \in \mathcal{L}$ there are declarative formulas $\alpha_{1}, \ldots, \alpha_{n} \in \mathcal{L}_{!}$, called the resolutions of $\varphi$, such that $\varphi \equiv \alpha_{1} \mathbb{\vee} \ldots \mathbb{V} \alpha_{n}$.

Having reviewed the main definitions and facts about $\operatorname{lnqB} \mathrm{B}^{\otimes}$, we can now delve into the issue of eliminability and definability of connectives in this system.

\section{Eliminability}

In this section we discuss which connectives from our language $\mathcal{L}$ are eliminable, and show that there is a unique minimal complete set of connectives for $\operatorname{lnq} \mathrm{B}^{\otimes}$. These results are straightforward adaptations to our language of results from [4], although we will lay out the consequences of these results more systematically. First, let us show that the connectives $\perp, \mathbb{V}$, and $\rightarrow$ are not eliminable: omitting any of these connectives from our language results in a loss of expressive power.

Proposition 5. $\perp$ is not eliminable.

Proof. We have to show that no $\perp$-free formula of $\mathcal{L}$ is equivalent to $\perp$. Let $w_{t}$ be the valuation function mapping all atomic formulas to 1 . A straightforward induction shows that every $\perp$-free formula is supported at the state $\left\{w_{t}\right\}$. Since $\left\{w_{t}\right\} \forall \perp$, it follows that no $\perp$-free formula is equivalent to $\perp$.

Proposition 6. $\mathbb{V}$ is not eliminable.

Proof. We must show that some formula of $\mathcal{L}$ is not equivalent to any $\mathbb{V}$-free formula. Consider the formula $? p:=p \Downarrow \neg p$. Proposition 3 ensures that every $\mathbb{V}$-free formula is truth-conditional. Since we saw above that $? p$ is not truthconditional, it follows that it is not equivalent to any $\mathbb{V}$-free formula.

Proposition 7. $\rightarrow$ is not eliminable. 
Proof. We show that $\top$ (defined as $\neg \perp$, i.e., $\perp \rightarrow \perp$ ) is not equivalent to any $\rightarrow$-free formula. Let $w_{f}$ be the valuation mapping all atomic formulas to 0 . A straightforward induction shows that no $\rightarrow$-free formula is supported at $\left\{w_{f}\right\}$. Since $T$ is supported at $\left\{w_{f}\right\}$, no $\rightarrow$-free formula is equivalent to $T$.

This shows that, starting from the repertoire of connectives $\{\perp, \wedge, \mathbb{V}, \rightarrow, \otimes\}$, none of $\perp, \mathbb{V}$, and $\rightarrow$ can be dropped without a loss in expressive power. We are now going to see that these three connectives together are sufficient to express anything that is expressible in $\operatorname{lnqB}{ }^{\otimes}$.

Proposition 8. $\{\perp, \rightarrow, \mathbb{V}\}$ is a complete set of connectives for InqB $B^{\otimes}$.

Proof. We need to show that for all $\varphi \in \mathcal{L}$ there is a $\varphi^{*} \in \mathcal{L}[\perp, \rightarrow, \mathbb{V}]$ such that $\varphi \equiv \varphi^{*}$. First, it follows from Proposition 4 and the fact that $\{\perp, \rightarrow\}$ is complete for classical propositional logic that any any declarative formula $\alpha \in \mathcal{L}[\perp, \wedge, \rightarrow, \otimes]$ is equivalent to a formula $\alpha^{*} \in \mathcal{L}[\perp, \rightarrow]$.

Now take any $\varphi \in \mathcal{L}$. By Theorem 1 we have $\varphi \equiv \alpha_{1} \mathbb{V} \ldots \mathbb{V} \alpha_{n}$ for some formulas $\alpha_{1}, \ldots, \alpha_{n} \in \mathcal{L}[\perp, \wedge, \rightarrow, \otimes]$. Now let $\varphi^{*}:=\alpha_{1}^{*} \mathbb{\vee} \ldots \mathbb{\vee} \alpha_{n}^{*}$, where $\alpha_{1}^{*}, \ldots, \alpha_{n}^{*}$ are defined as above. Since $\alpha_{i} \equiv \alpha_{i}^{*}$ for $i \leq n$, we have $\varphi \equiv \varphi^{*}$. And since $\alpha_{i}^{*} \in \mathcal{L}[\perp, \rightarrow]$ for $i \leq n$, we have $\varphi^{*} \in \mathcal{L}[\perp, \rightarrow, \mathbb{V}]$.

Corollary 1. The set of connectives $\{\wedge, \otimes\}$ is eliminable in InqB $B^{\otimes}$. In particular, both $\wedge$ and $\otimes$ are eliminable.

Proposition 8 together with the non-eliminability of $\perp, \rightarrow$, and $\mathbb{\vee}$ implies that Inq $B^{\otimes}$ admits only one minimal complete set of connectives, namely, $\{\perp, \rightarrow, \mathbb{V}\}$.

Theorem 2. $\{\perp, \rightarrow, \mathbb{V}\}$ is the only minimal complete set for Inq $B^{\otimes}$.

Proof. It follows from Propositions 5, 6, and 7 that any complete set of connectives $C$ for $\operatorname{InqB}^{\otimes}$ must include $\{\perp, \rightarrow, \mathbb{V}\}$. Since $\{\perp, \rightarrow, \mathbb{V}\}$ is itself a complete set, it is the only minimal one.

We have thus achieved a complete characterization of:

- which connectives are eliminable in $\operatorname{lnqB}^{\otimes}(\wedge$ and $\otimes$, but not $\perp, \rightarrow$ and $\mathbb{V})$;

- which sets of connectives are complete (those which include $\{\perp, \rightarrow, \mathbb{V}\}$ );

- which sets of connectives are minimal complete (only $\{\perp, \rightarrow, \mathbb{V}\}$ ).

\section{Independence of the connectives}

In this section, we turn to the issue of definability. The main contribution of the paper is to answer the following question, which is open in the literature: which connectives of $\operatorname{InqB}^{\otimes}$ are definable in terms of the remaining connectives? We will see that the answer is none: thus, although $\{\perp, \wedge, \mathbb{V}, \rightarrow, \otimes\}$ is not a minimal set of connectives for $\operatorname{lnqB} B^{\otimes}$, it is an independent set of connectives.

For a start, notice that the question of definability only arises for $\wedge$ and $\otimes$ : as we saw, the connectives $\perp, \mathbb{V}$, and $\rightarrow$ are not even eliminable in InqB ${ }^{\otimes}$, which a fortiori implies that they are not definable. We will examine first the case of tensor disjunction, and then the case of conjunction. 


\subsection{Undefinability of $\otimes$}

Fix three propositional letters $q_{1}, q_{2}, q_{3}$ and consider:

$-\psi:=q_{1} \mathbb{\vee} q_{2} \mathbb{\vee} q_{3}$

$-s:=\left\{w_{1}, w_{2}, w_{3}\right\}$ where $w_{i}\left(q_{i}\right)=1$ and $w_{i}(r)=0$ for all $r \neq q_{i}$

Lemma 1. For any context $\varphi(p)$, with $\varphi \in \mathcal{L}[\perp, \wedge, \rightarrow, \mathbb{V}]$ not containing $q_{1}, q_{2}, q_{3}$, one of the following holds:

$$
\varphi(\psi) \equiv_{s} \top \quad \varphi(\psi) \equiv_{s} \perp \quad \varphi(\psi) \equiv_{s} \psi
$$

Proof. We proceed by induction on $\varphi$. To ease notation, given a formula $\chi(p)$, let us write $\chi^{\star}$ for $\chi(\psi)$.

$-\varphi=p$ or $\varphi=\perp$. Then $\varphi^{\star}$ is either $\psi$ or $\perp$, and we are done.

$-\varphi=r$ for an atomic formula $r$ different from $p, q_{1}, q_{2}, q_{3}$. Then $\varphi^{\star}=r$. Since $r$ is false at all worlds in $s$ we have $r \equiv_{s} \perp$.

$-\varphi=\eta \wedge \theta$. Then $\varphi^{\star}=\eta^{\star} \wedge \theta^{\star}$. By induction hypothesis, each of $\eta^{\star}$ and $\theta^{\star}$ is equivalent modulo $s$ to either one of $T, \perp, \psi$. Since the conjunction of any two formulas from $\{T, \perp, \psi\}$ is logically equivalent to a formula from this set, we can conclude that $\varphi^{\star}$ is equivalent modulo $s$ either to $\top, \perp$ or $\psi$.

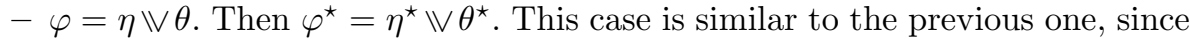
the inquisitive disjunction of any two formulas from $\{\top, \perp, \psi\}$ is logically equivalent to a formula from this set.

$-\varphi=\eta \rightarrow \theta$. Then $\varphi^{\star}=\eta^{\star} \rightarrow \theta^{\star}$. We consider three cases:

- $\eta^{\star} \equiv_{s} \top$. In this case, by persistency we have $\varphi^{\star} \equiv_{s} \top \rightarrow \theta^{\star} \equiv \theta^{\star}$. By induction hypothesis, $\theta^{\star}$ is equivalent in $s$ to $\top, \perp$, or $\psi$, so we are done.

- $\eta^{\star} \equiv_{s} \perp$. In this case, by the empty state property $\varphi^{\star} \equiv_{s} \perp \rightarrow \theta^{\star} \equiv \top$.

- $\eta^{\star} \equiv_{s} \psi$. In this case, we need to distinguish three sub-cases:

$* \theta^{\star} \equiv_{s} \top$. In this case, $\varphi^{\star} \equiv_{s} \psi \rightarrow \top \equiv \top$.

$* \theta^{\star} \equiv_{s} \psi$. In this case, $\varphi^{\star} \equiv_{s} \psi \rightarrow \psi \equiv \top$.

$* \theta^{\star} \equiv_{s} \perp$. In this case $\varphi^{\star} \equiv_{s} \psi \rightarrow \perp$. We claim that $\psi \rightarrow \perp \equiv_{s} \perp$. We need to show that the only $t \subseteq s$ that supports $\psi \rightarrow \perp$ is $\emptyset$. Notice that, by the definitions of $\psi$ and negation, $\psi \rightarrow \perp=\neg\left(q_{1} \mathbb{\vee} q_{2} \mathbb{\vee} q_{3}\right)$. Suppose for a contradiction that $\emptyset \neq t \subseteq s$ and $t \models \neg\left(q_{1} \mathbb{\vee} q_{2} \mathbb{\vee} q_{3}\right)$. Take a valuation $w_{i} \in t$. By persistency, $\left\{w_{i}\right\} \models \neg\left(q_{1} \mathbb{\vee} q_{2} \mathbb{\vee} q_{3}\right)$. But this is impossible, since by construction $\left\{w_{i}\right\} \models q_{i}$.

Now just observe the support conditions for the formulas $\psi$ and $\psi \otimes \psi$ relativized to the given state $s$. Letting \#t be the cardinality of $t$, for all $t \subseteq s$ we have:

$-t=\psi \Longleftrightarrow \# t \leq 1$

$-t=\psi \otimes \psi \Longleftrightarrow \# t \leq 2$

This means that we have:

$$
\psi \otimes \psi \not_{s} \top \quad \psi \otimes \psi \not \equiv_{s} \perp \quad \psi \otimes \psi \not \equiv_{s} \psi
$$

From this observation and Lemma 1, we get the following proposition. 
Proposition 9. For all $\varphi(p) \in \mathcal{L}[\perp, \wedge, \rightarrow, \mathbb{V}]$ which do not contain $q_{1}, q_{2}, q_{3}$ :

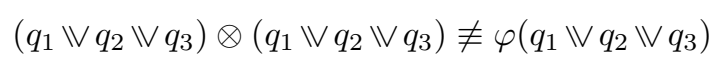

It is now easy to get the desired undefinability result.

Theorem 3. $\otimes$ is not definable from $\{\perp, \wedge, \rightarrow, \mathbb{V}\}$.

Proof. Consider a context $\varphi\left(p_{1}, p_{2}\right)$ with $\varphi \in \mathcal{L}[\perp, \wedge, \rightarrow, \mathbb{V}]$ (possibly containing other atoms besides $p_{1}, p_{2}$ ). Let $q_{1}, q_{2}, q_{3}$ be three atoms which do not occur in $\varphi$ and let $\psi=q_{1} \Downarrow q_{2} \Vdash q_{3}$. By Proposition 9 we have:

$$
\psi \otimes \psi \not \equiv \varphi(\psi, \psi)
$$

which implies that $\varphi\left(p_{1}, p_{2}\right)$ does not define $\otimes$.

\subsection{Undefinability of $\wedge$}

We will follow a strategy similar to that we used for $\otimes$. Fix two atomic formulas $q_{1}, q_{2}$, and let $s=\left\{w_{12}, w_{1}, w_{2}\right\}$ where $w_{12}$ makes both $q_{1}$ and $q_{2}$ true, $w_{1}$ makes only $q_{1}$ true, and $w_{2}$ makes only $q_{2}$ true. Moreover, suppose that all atoms different from $q_{1}, q_{2}$ are false at all three worlds. We will show the following.

Lemma 2. Take a context $\varphi\left(p_{1}, p_{2}\right)$, with $\varphi \in \mathcal{L}[\perp, \rightarrow, \mathbb{V}, \otimes]$ not containing $q_{1}, q_{2}$. Then one of the following claims holds:

1. $\varphi\left(? q_{1}, ? q_{2}\right) \equiv_{s} \perp$

2. $\varphi\left(? q_{1}, ? q_{2}\right)$ is supported by all subsets of $s$ of cardinality 1 , and by at least one subset of $s$ of cardinality 2 .

Proof. We proceed by induction on $\varphi$. To ease notation, given a context $\chi\left(p_{1}, p_{2}\right)$, let us write $\chi^{\star}$ for $\chi\left(? q_{1}, ? q_{2}\right)$.

$-\varphi=p_{1}$. Then $\varphi^{\star}=? q_{1}$ is supported by all singleton states, and also by the state $\left\{w_{12}, w_{1}\right\} \subseteq s$, which has cardinality 2 . So claim 2 holds.

$-\varphi=p_{2}$. Analogous.

$-\varphi=\perp$. Then $\varphi^{\star}=\perp$, so claim 1 holds.

$-\varphi=r$ for $r \neq p_{1}, p_{2}, q_{1}, q_{2}$. Then $\varphi^{\star}=r$. Since $r$ is false in all worlds in $s$ we have $r \equiv_{s} \perp$, so claim 1 holds.

$-\varphi=\eta \rightarrow \theta$. We distinguish three cases:

- $\eta^{\star} \equiv_{s} \perp \equiv_{s} \theta^{\star}$. Then $\varphi^{\star} \equiv_{s} \perp \rightarrow \perp=\top$, therefore claim 2 holds.

- $\eta^{\star} \not_{s} \perp \equiv_{s} \theta^{\star}$. Then $\varphi^{\star} \equiv_{s} \eta^{\star} \rightarrow \perp$. We will show that $\varphi^{\star} \equiv_{s} \perp$. Take any non-empty $t \subseteq s$, and let $\{w\} \subseteq t$. By the induction hypothesis on $\eta$ we have $\{w\} \mid=\eta^{\star}$, but $\{w\} \mid \not \perp$. Therefore, $t \not \models \eta^{\star} \rightarrow \perp$. Thus, $\varphi^{\star}$ is not supported at any non-empty subset of $s$, which means that $\varphi^{\star} \equiv_{s} \perp$.

- $\theta^{\star} \not \equiv_{s} \perp$. Then by induction hypothesis $\theta^{\star}$ is supported by all singleton substates of $s$, and also by a substate of $s$ of cardinality 2. Since any state that supports $\theta^{\star}$ also supports $\varphi^{\star}=\eta^{\star} \rightarrow \theta^{\star}$, by persistency, claim 2 holds for $\varphi^{\star}$. 


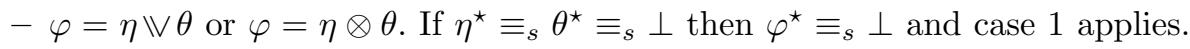
Otherwise, at least one of $\eta^{\star}$ and $\theta^{\star}$ is not $s$-equivalent to $\perp$. Suppose it is $\eta^{\star}$. Then by induction hypothesis $\eta^{\star}$ is supported by all singleton substates of $s$, and by a substate of $s$ of cardinality 2. Since any state that supports $\eta^{\star}$ also supports $\eta^{\star} \mathbb{V} \theta^{\star}$ as well as $\eta^{\star} \otimes \theta^{\star}$, claim 2 applies to $\varphi^{\star}$.

Now consider the formula ? $q_{1} \wedge ? q_{2}$. For all $t \subseteq s$ we have:

$-t=? q_{1} \wedge ? q_{2} \Longleftrightarrow \# t \leq 1$

Thus, ? $q_{1} \wedge ? q_{2} \not \equiv_{s} \perp$, and moreover $? q_{1} \wedge ? q_{2}$ is not supported by any substate of $s$ of cardinality 2 . It follows from Lemma 2 that $? q_{1} \wedge ? q_{2} \not \equiv_{s} \varphi\left(? q_{1}, ? q_{2}\right)$ for any context $\varphi\left(p_{1}, p_{2}\right)$ which does not contain the atoms $q_{1}, q_{2}$. Since logical equivalence implies equivalence in every state, we obtain the following proposition.

Proposition 10. For all $\varphi\left(p_{1}, p_{2}\right) \in \mathcal{L}[\perp, \rightarrow, \mathbb{V}, \otimes]$ which do not contain $q_{1}, q_{2}$ :

$$
? q_{1} \wedge ? q_{2} \not \equiv \varphi\left(? q_{1}, ? q_{2}\right)
$$

This yields as a corollary the undefinability of $\wedge$.

Theorem 4. $\wedge$ is not definable from $\{\perp, \rightarrow, \mathbb{V}, \otimes\}$.

Proof. Take a context $\varphi\left(p_{1}, p_{2}\right)$, where $\varphi \in \mathcal{L}[\perp, \rightarrow, \mathbb{V}, \otimes]$ (possibly containing other atoms besides $p_{1}, p_{2}$ ). Let $q_{1}, q_{2}$ be two atoms that do not occur in $\varphi$. By Proposition 10 we have $? q_{1} \wedge ? q_{2} \not \equiv \varphi\left(? q_{1}, ? q_{2}\right)$, which implies that $\varphi\left(p_{1}, p_{2}\right)$ does not define $\wedge$.

This completes our study of definability in $\operatorname{lnq} \mathrm{B}^{\otimes}$ : no connective in $\operatorname{lnq} \mathrm{B}^{\otimes}$ is definable in terms of the others; although the set of connectives $\{\perp, \wedge, \mathbb{V}, \rightarrow, \otimes\}$ is not a minimal complete set for $\operatorname{lnq} B^{\otimes}$, it is an independent set.

\section{Conclusion}

We have studied connectives in inquisitive logic enriched with tensor disjunction. We have shown that, starting from the set of primitives $\{\perp, \wedge, \rightarrow, \mathbb{V}, \otimes\}$, the only minimal complete subset is $\{\perp, \rightarrow, \vee\}$. Thus, $\wedge$ and $\otimes$ do not add to the expressive power of the language. However, the set $\{\perp, \wedge, \rightarrow, \mathbb{V}, \otimes\}$ is independent, i.e., no connective is definable in terms of the others. The undefinability results for $\wedge$ and $\otimes$ use nontrivial combinatorial arguments. The result for $\otimes$ answers a question raised in [6] and [27]. It is worth pointing out that our proof establishes something slightly stronger than undefinability. Undefinability amounts to:

For every putative definition $\varphi(p, q)$ of connective $\circ$, there are formulas $\psi_{\varphi}, \chi_{\varphi}$ such that $\varphi\left(\psi_{\varphi}, \chi_{\varphi}\right) \not \equiv \psi_{\varphi} \circ \chi_{\varphi}$.

However, our proofs for $\otimes$ and $\wedge$ establish results of the following form:

There are $\psi, \chi$ such that, for every putative definition $\varphi(p, q)$ of $\circ$, $\varphi\left(\psi^{\prime}, \chi^{\prime}\right) \not \equiv \psi^{\prime} \circ \chi^{\prime}$, where $\psi^{\prime}, \chi^{\prime}$ differ from $\psi, \chi$ by a renaming of atoms.

In future work, it would be interesting to extend our study of expressiveness and definability to a setting where the operators $\neg$ and ? are taken as primitives. 


\section{References}

1. Abramsky, S., Väänänen, J.: From IF to BI. Synthese 167(2), 207-230 (2009)

2. Baltag, A., Moss, L.S., Solecki, S.: The logic of public announcements, common knowledge, and private suspicions. In: Proceedings of the 7th Conference on Theoretical Aspects of Rationality and Knowledge. pp. 43-56. Morgan Kaufmann Publishers (1998)

3. Bledin, J.: Logic informed. Mind 123(490), 277-316 (2014)

4. Ciardelli, I.: Inquisitive semantics and intermediate logics (2009), MSc Thesis, University of Amsterdam

5. Ciardelli, I.: Dependency as question entailment. In: Abramsky, S., Kontinen, J., Väänänen, J., Vollmer, H. (eds.) Dependence Logic: theory and applications, pp. 129-181. Springer International Publishing Switzerland (2016)

6. Ciardelli, I.: Questions in logic. Ph.D. thesis, Institute for Logic, Language and Computation, University of Amsterdam (2016)

7. Ciardelli, I.: Questions as information types. Synthese 195, 321-365 (2018). https://doi.org/doi:10.1007/s11229-016-1221-y

8. Ciardelli, I., Roelofsen, F.: Inquisitive logic. Journal of Philosophical Logic 40(1), 55-94 (2011)

9. Dekker, P.: Transsentential Meditations. Ups and Downs in Dynamic Semantics. Ph.D. thesis, ILLC, University of Amsterdam (1993)

10. van Ditmarsch, H., van der Hoek, W., Kooi, B.: Dynamic Epistemic Logic. Springer (2007)

11. Galliani, P.: Inclusion and exclusion dependencies in team semantics - on some logics of imperfect information. Annals of Pure and Applied Logic 163(1), 68-84 (2012)

12. Galliani, P.: Epistemic operators in dependence logic. Studia Logica 101(2), 367397 (2013)

13. Goranko, V., Kuusisto, A.: Logics for propositional determinacy and independence. The Review of Symbolic Logic 11(3), 470-506 (2018)

14. Grilletti, G.: Disjunction and existence properties in inquisitive first-order logic. Studia Logica pp. 1-36 (2017)

15. Groenendijk, J., Stokhof, M., Veltman, F.: Coreference and modality in the context of multi-speaker discourse. In: Kamp, H., Partee, B.H. (eds.) Context Dependence in the Analysis of Linguistic Meaning, pp. 195-216. IMS (1997)

16. Kolodny, N., MacFarlane, J.: Ifs and oughts. The Journal of philosophy 107(3), 115-143 (2010)

17. Kontinen, J., Väänänen, J.: On definability in dependence logic. Journal of Logic, Language and Information 18(3), 317-332 (2009)

18. McKinsey, J.C.C.: Proof of the independence of the primitive symbols of heyting's calculus of propositions. The Journal of Symbolic Logic 4(4), 155-158 (1939), http://www.jstor.org/stable/2268715

19. Plaza, J.: Logics of public communications. In: Emrich, M., Pfeifer, M., Hadzikadic, M., Ras, Z. (eds.) Proceedings of the Fourth International Symposium on Methodologies for Intelligent Systems. pp. 201-216. Oak Ridge National Laboratory (1989), reprinted as [?]

20. Punčochár, V.: Weak negation in inquisitive semantics. Journal of Logic, Language, and Information 24(3), 323-355 (2015)

21. Roelofsen, F.: Algebraic foundations for the semantic treatment of inquisitive content. Synthese 190(1), 79-102 (2013). https://doi.org/10.1007/s11229-013-0282-4 
22. Väänänen, J.: Dependence Logic: A New Approach to Independence Friendly Logic. Cambridge University Press (2007)

23. Veltman, F.: Data semantics. In: Groenendijk, J., Janssen, T., Stokhof, M. (eds.) Formal Methods in the Study of Language. Mathematical Centre (1981)

24. Veltman, F.: Defaults in update semantics. Journal of Philosophical Logic 25(3), 221-261 (1996)

25. Yalcin, S.: Epistemic modals. Mind 116(464), 983-1026 (2007)

26. Yang, F.: On extensions and variants of dependence logic: A study of intuitionistic connectives in the team semantics setting. Ph.D. thesis, University of Helsinki (2014)

27. Yang, F.: Uniform definability in propositional dependence logic. The Review of Symbolic Logic 10(1), 65-79 (2017)

28. Yang, F., Väänänen, J.: Propositional logics of dependence. Annals of Pure and Applied Logic 167(7), 557-589 (2016) 